\title{
SNS SRF TIME DEPENDENT CAVITY RF RESONANCE SHIFT DUE TO LORENTZ FORCE INDUCED MECHANICAL EXCITATION*
}

\author{
Stephen Ellis ${ }^{\dagger}$, Los Alamos National Laboratory \\ Los Alamos, New Mexico 87545, USA
}

\begin{abstract}
The Spallation Neutron Source (SNS) accelerator incorporates a Superconducting RF (SRF) accelerator for the final section of the three-section pulsed mode linac. The SRF linac cavities are of conventional thin-walled niobium construction. Dynamic detuning of the SRF cavities due to Lorentz force induced mechanical excitation is a critical concern in the design of such cavities. The pulsed RF induces cyclic Lorentz forces on the cavity surface that are proportional to the rising and falling surface magnetic and electric fields. The induced Lorentz force levels are small but will mechanically excite a thin-walled structurally compliant cavity. A FORTRAN code has been developed that calculates the time varying cavity frequency shift due to the cyclic Lorentz force excitation. The code generates an ABAQUS [1] finite element mesh representing the cavity geometry and applies calculated Lorentz forces to the mesh utilizing cavity SUPERFISH [2] results as input. ABAQUS is then utilized to calculate the deformed cavity shape due to the dynamic excitation, using very small time steps. The ABAQUS solver accounts for the time dependency of the Lorentz forces, inertial effects, material damping, and structural boundary conditions. An algorithm incorporating the Slater-perturbation theory is subsequently utilized to calculate the resonant frequency shift for the deformed cavity shape at every time increment.
\end{abstract}

\section{SRF DESCRIPTION AND OPERATION}

The SRF provides final acceleration of the $26-\mathrm{mA} \mathrm{H}^{-}$ beam from $186-\mathrm{MeV}$ to $1-\mathrm{GeV}$. The SRF operates in $\pi$ mode at $805-\mathrm{MHz}$. The linac consists of 81 six-cell elliptical cavities. Each cavity is housed within its own helium vessel, which in turn, is housed in sets of three or four per cryostat within 33 cryostats. The cavities are of conventional axisymmetric thin-walled design as shown in Figure 1.

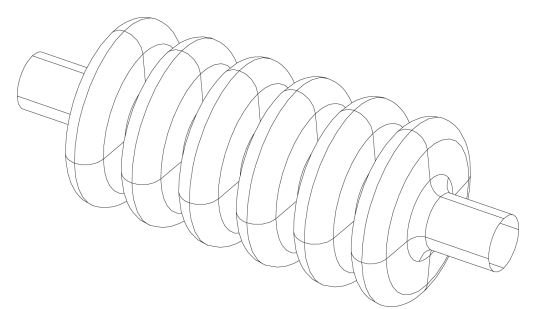

Figure 1: Six-cell SRF Cavity

\footnotetext{
* Work supported by the Office of Basic Energy Science, Office of Science of the US Department of Energy, and by Oak Ridge National Laboratory.

†ellis@lanl.gov
}

The linac utilizes only two cavity configurations, a medium beta and a high beta design. General information for the two designs is listed in Table 1.

Table 1: Cavity Design Summary [3]

\begin{tabular}{|c|c|c|}
\hline Property & Medium Beta & High Beta \\
\hline Geometric beta & $61 \%$ & $81 \%$ \\
\hline Cavity quantity & 33 & 48 \\
\hline $\mathrm{E}_{0}$ & $13.4-16.4-\mathrm{MV} / \mathrm{m}$ & $17.9-24.4-\mathrm{MV} / \mathrm{m}$ \\
\hline $\begin{array}{c}\text { Max. surface } \\
\text { E field }\end{array}$ & $27.5-\mathrm{MV} / \mathrm{m}$ & $35-\mathrm{MV} / \mathrm{m}$ \\
\hline Physical length & $107-\mathrm{cm}$ & $129-\mathrm{cm}$ \\
\hline Bore diameter & $8.6-\mathrm{cm}$ & $9.7-\mathrm{cm}$ \\
\hline
\end{tabular}

The cavities are fabricated of thin walled 4-mm thick niobium sheet. Individual cell halves are stamped, trimmed, and subsequently electron-beam welded together forming complete cavity assemblies. The SNS SRF cavities are unique in that the two end cells are slightly different geometrically than the four center cells. After forming and etching operations, the final nominal cavity wall thickness is approximately $3.8-\mathrm{mm}$. High purity niobium is utilized for its cryogenic superconducting qualities. Typical room temperature niobium mechanical properties are listed in Table 2. Fabrication drawings for the medium and high beta cavities are listed in references [4] and [5] respectively.

Table 2: Niobium Mechanical Properties

\begin{tabular}{|c|c|}
\hline Property & Value \\
\hline Young's modulus & $14.2 \mathrm{E} 6-\mathrm{psi}$ \\
\hline Poisson's ratio & 0.38 \\
\hline Density & $0.31-\mathrm{lb}_{\mathrm{f}} / \mathrm{in}^{3}$ \\
\hline
\end{tabular}

Each cavity is installed within a helium vessel, the endwalls of which provide structural support for the cavity. Structural attachment to the cavity is accomplished by means of interface flanging midway along the tube sections at either cavity end. A tuning device is incorporated at one end allowing axially extension or compression of the cavity in order to provide an electromagnetic tuning means during operation. No other structural support is provided.

The SNS linac is a pulsed machine, i.e. particle acceleration and thus introduction of RF power into the accelerator is not continuous. The RF power is energized for a $1250-\mu$ s duration pulse sixty times per second yielding an RF duty factor of $7.0 \%$. 


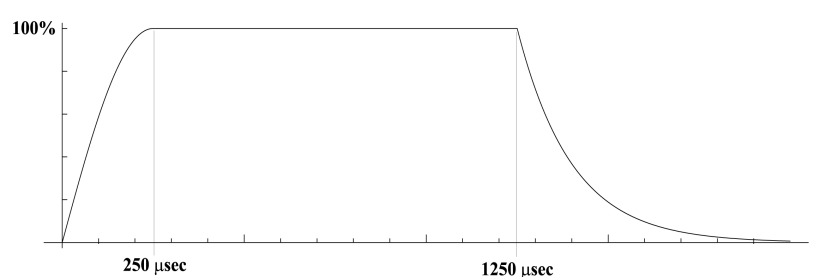

Figure 2: RF Pulse Profile

The pulse initiates with a sinusoidal ramp during the first $250-\mu \mathrm{s}$, dwells for 1-ms at full power and then decays exponentially with an approximate time constant of $150-\mu \mathrm{s}$. The pulse profile is plotted in Figure 2.

\section{LORENTZ FORCE DETUNING}

The electric and magnetic fields present on the cavity surface induce small surface normal forces known as Lorentz forces. These pressures are proportional to the surface electric and magnetic fields as described below [6].

$$
P=\frac{1}{2}\left(\mu_{0}|H|^{2}-\varepsilon_{0}|\mathbf{E}|^{2}\right)
$$

The forces act inward near the cell equator and outward near the cell bore. The electric and magnetic fields and thus the induced Lorentz forces are axisymmetric with respect to the cavity axis. The calculated maximum Lorentz forces for the medium beta cavity are 580-Pa acting outward near the cell equator and $1.65-\mathrm{KPa}$ acting inward at the cell bore. The calculated maximum Lorentz forces for the high beta cavity are $1.01-\mathrm{KPa}$ acting outward near the cell equator and $2.58-\mathrm{KPa}$ acting inward at the cell bore. The forces are significantly higher for the high beta cavity because of its significantly higher $\mathrm{E}_{0}$.

The Lorentz surface pressures, although small in magnitude are proportional to the surface electric and magnetic fields and thus rise and fall as the RF power rises and falls during each pulse. A critical concern is dynamic excitation and consequent electromagnetic detuning and field distortion of the cavities by the cyclic Lorentz forces. Excessive detuning will impede or prevent proper RF power coupling to the cavity.

\section{COUPLED STRUCTURAL AND ELECTROMAGNETIC MODELING}

Of interest are the time dependant changes in the cavity electromagnetic performance, for example resonant frequency and field distribution, due to cyclic Lorentz force induced dynamic deformation. A FORTRAN code has been written which provides links between SUPERFISH, an electromagnetic solver, and ABAQUS, a structural finite element code, permitting accurate modeling of coupled structural and electromagnetic behavior.

The code creates an input file for ABAQUS from a SUPERFISH cavity solution file. The code generates an axisymmetric finite element mesh complete with accurate discrete Lorentz pressure loads at each node of the finite element mesh. The code also has the ability to construct elements representing external ring stiffeners if incorporated within the cavity design. A sample finite element mesh is depicted in Figure 3. Boundary conditions representing structural constraints as well as spring elements representing the axial tuner are readily applied to the finite element mesh.

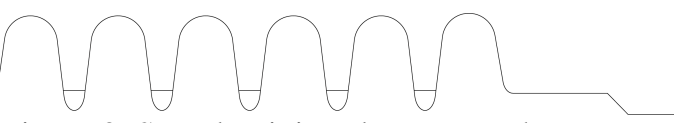

Figure 3: Sample Finite Element Mesh

ABAQUS is then used to calculate the deformed cavity shape due to the dynamic excitation. These calculations are performed incrementally, using very small time steps, in order to accurately integrate time dependant parameters. The ABAQUS solver accounts for the time dependency of the Lorentz forces, inertial effects, material damping, and the applied structural boundary conditions.

The calculated deformed cavity shape at any time increment may be captured and utilized as input for SUPERFISH allowing complete evaluation of cavity electromagnetic performance. A separate code has been written which calculates the cavity frequency shift at every time step utilizing the Slater-perturbation theory directly from the ABAQUS output.

\section{SNS MEDIUM BETA AND HIGH BETA CAVITY MODELING RESULTS}

Calculations as described above have been carried out for cavities approximating the geometry and electromagnetic performance of the present SNS cavity designs. Figures 4 and 5 are plots of calculated cavity resonance frequency shift for the initial 60-ms of SRF operation. Note that different scales have been used for the vertical axes. This time period includes four RF pulses. A time increment of $60-\mu$ s was utilized for these calculations.

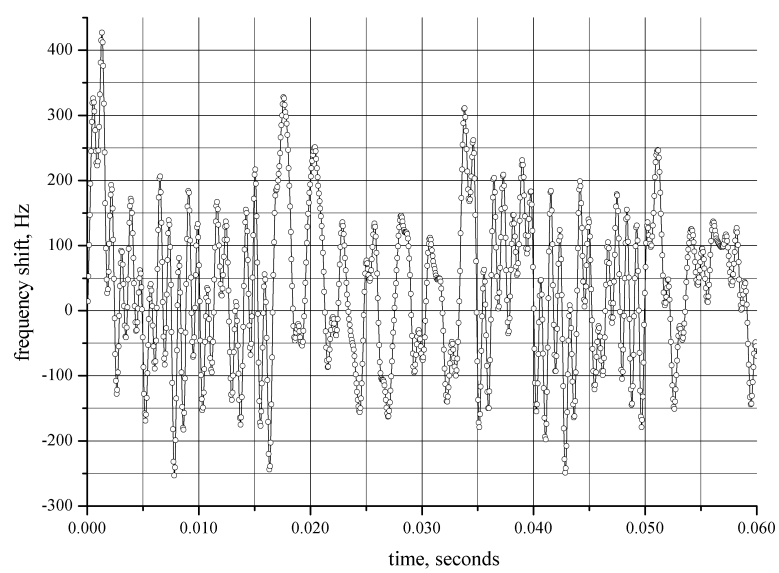

Figure 4: Calculated Dynamic Frequency Shift Values for the Medium Beta Cavity

Mechanical ringing of the cavity and the corresponding resonant electromagnetic frequency is apparent in the 
plots. Spikes in the frequency shift values at $16.67-\mathrm{ms}$ intervals are obvious, corresponding to the $60-\mathrm{Hz} \mathrm{RF}$ pulsing frequency. Spectral analysis detected significant frequency content at the $60-\mathrm{Hz}$ RF pulsing frequency and additionally at many frequencies that correspond to axial mechanical modes of the particular cavity. The axial mechanical modes for each cavity were calculated for verification independently with ABAQUS.

The effects of mechanical damping were not included in the calculations. Damping for a welded structure of this nature is very small and thus will have minimal impact on dynamic response. The calculations are based on room temperature mechanical properties. A slight increase of Young's modulus at the cryogenic operating temperature will increase the cavity's mechanical resonant frequencies a small amount and thus correspondingly affect dynamic response.

The calculated response for the medium beta cavity has an average shift or dc component of $35-\mathrm{Hz}$ for the $60-\mathrm{ms}$ time increment studied. The calculated shift values vary by about $400-\mathrm{Hz}$ maximum from the average shift value. The calculated static or non-pulsed RF frequency shift due to the Lorentz force loading is $380-\mathrm{Hz}$.

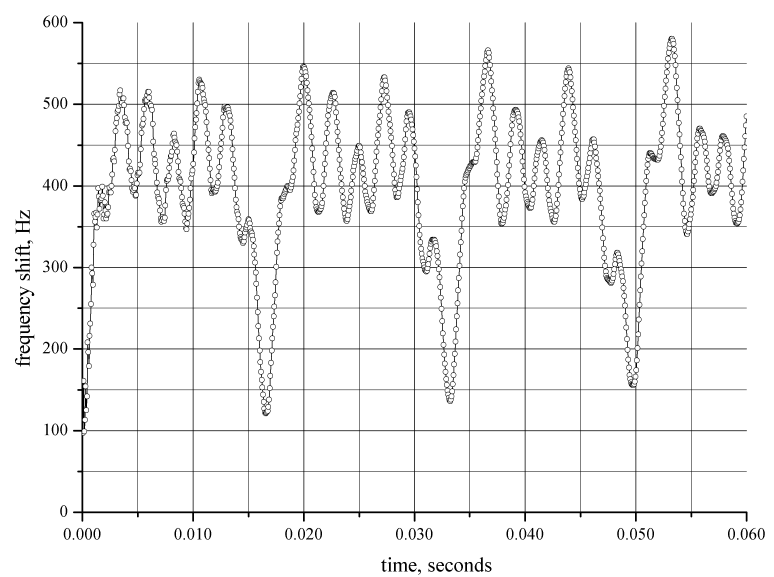

Figure 5: Calculated Dynamic Frequency Shift Values for the High Beta Cavity

The calculated response for the high beta cavity has a much larger average shift value of $400-\mathrm{Hz}$ for the $60-\mathrm{ms}$ time increment studied. The calculated shift values vary by about $300-\mathrm{Hz}$ maximum from the nominal shift value. The calculated RF frequency shift due to the static Lorentz force loading is $397-\mathrm{Hz}$ for the high beta cavity.

The high beta cavity results show lower high frequency content when compared to the medium beta results. This is probably due to the particular axial mechanical modes and their respective coupling levels that are excited by the $60-\mathrm{Hz}$ Lorentz force cyclic loading.

The results for the high beta cavity also show slight amplitude gain during the $60-\mathrm{ms}$ time increment. This was not evident with the medium beta cavity results. This is probably due to the closer proximity of the fundamental cavity axial mechanical mode frequency to the RF pulsing frequency. This anomaly should be evaluated further. Mechanical modifications may be necessary to reduce the coupling between the RF pulsing excitation and mechanical modes.

Further calculations should be performed for longer time increments to fully evaluate cavity's long-term dynamic performance. The $60-\mathrm{ms}$ time increment utilized for these studies can miss lower frequency behavior that may be important.

Accurate representation of the cavity structural boundaries is also important. Flexibility in the helium vessel end wall will affect cavity dynamic performance and the response to the cyclic Lorentz forces. Likewise, accurate structural characterization of the cavity tuner is also important.

The tools described above are very useful in evaluating cavity mechanical tuning schemes. Necessary tuner loads and the corresponding cavity deformation can be easily calculated, in addition to the resulting cavity electromagnetic performance. The tools are also valuable in optimizing cavity geometry, wall thickness and stiffener ring geometry.

\section{REFERENCES}

[1] ABAQUS, Version 6.2.1, Hibbitt, Karlsson \& Sorenson, Inc. (2001)

[2] James H. Billen and Lloyd M. Young, "POISSON SUPERFISH", LANL report LA-UR-96-1834 (revised May 2, 2001)

[3] "SNS Parameters List", SNS-100000000-PL0001-R05 (May 2001)

[4] "SNS 0.61 Beta Cryo-Module Assembly", Jefferson Lab drawing CRM9007010-0000 (May 15, 2001)

[5] "SNS 0.81 Beta Cryo-Module Assembly", Jefferson Lab drawing CRM9007010-0002 (February 27, 2000)

[6] E. Haebel and J. Tuckmantel, "Electromagnetic Surface Forces in RF Cavities", CERN-AT-RF(Int)91-99 (December 11, 1991) 